\title{
Prescribed Performance Fuzzy Adaptive Output-Feedback Control for Nonlinear Stochastic Systems
}

\author{
Lili Zhang, Shuai Sui, and Shaocheng Tong \\ Department of Basic Mathematics, Liaoning University of Technology, Jinzhou, Liaoning 121000, China \\ Correspondence should be addressed to Lili Zhang; lutzhanglili@163.com
}

Received 4 January 2014; Accepted 4 February 2014; Published 12 March 2014

Academic Editor: Ming Liu

Copyright (C) 2014 Lili Zhang et al. This is an open access article distributed under the Creative Commons Attribution License, which permits unrestricted use, distribution, and reproduction in any medium, provided the original work is properly cited.

\begin{abstract}
A prescribed performance fuzzy adaptive output-feedback control approach is proposed for a class of single-input and single-output nonlinear stochastic systems with unmeasured states. Fuzzy logic systems are used to identify the unknown nonlinear system, and a fuzzy state observer is designed for estimating the unmeasured states. Based on the backstepping recursive design technique and the predefined performance technique, a new fuzzy adaptive output-feedback control method is developed. It is shown that all the signals of the resulting closed-loop system are bounded in probability and the tracking error remains an adjustable neighborhood of the origin with the prescribed performance bounds. A simulation example is provided to show the effectiveness of the proposed approach.
\end{abstract}

\section{Introduction}

In the past decade, control design and stability analysis on stochastic systems have received considerable attention, since stochastic modeling has come to play an important role in many real systems, including nuclear processes, thermal processes, chemical processes, biology, socioeconomics, and immunology [1-4]. Especially, the investigations on the control design methods of nonlinear stochastic systems have received more attention in recent years based on backstepping technique. For example, the adaptive backstepping control problem has been investigated in [5] for a class of SISO strict-feedback stochastic systems by a risk-sensitive cost criterion. An output-feedback stabilization method has been proposed for a class of strict-feedback stochastic nonlinear systems by using the quartic Lyapunov function in [6]. Two backstepping control design approaches have been developed for nonlinear stochastic systems with the Markovian switching in $[7,8]$. By using a linear reduced-order state observer, several different output-feedback controllers have been developed for strict-feedback nonlinear stochastic systems with unmeasured states, such as tracking control [9], decentralized control [10], and time-delay systems [11]. However, these proposed control methods are only suitable for those nonlinear stochastic systems with nonlinear dynamic models known exactly or with the unknown parameters appearing linearly with respect to known nonlinear functions. To cope with the problems that the nonlinear dynamic models are unknown or the system uncertainties are not linearly parameterized, the adaptive output-feedback control approaches have been proposed for a class of uncertain nonlinear stochastic systems by using neural networks in $[12,13]$. The decentralized adaptive neural networks control methods have been developed in $[14,15]$ for a class of uncertain large-scale nonlinear stochastic systems on the basis of $[12,13]$.

Although the adaptive neural networks backstepping control approaches in [12-15] can solve the problem of the unmeasured states by designing a linear state observer, there is a limit; that is, uncertain terms are only the functions of the output of the controlled systems, not related to the other states variables. To solve this limit, some adaptive fuzzy output feedback control methods have been proposed for a class of nonlinear stochastic systems by designing nonlinear fuzzy state observers in [16-18].

It should be mentioned that the control methods [1218] can only solve output-feedback stabilization problem and cannot solve the output feedback tracking control problem. 
In addition, the tracking performance in the above control methods confined to converge to a small residual set, whose size depends on the design parameters and some unknown bounded terms; they cannot offer the guaranteed transient performance at time instants. As we know, the practical engineering often requires the proposed control scheme to satisfy certain quality of the performance indices, such as overshoot, convergence rate, and steady-state error. Prescribed performance issues are extremely challenging and difficult to be achieved, even in the case of the nonlinear behavior of the system in the presence of unknown uncertainties and external disturbances. More recently, a design solution called prescribed performance control for the problem has been proposed in [19] for a class of feedback linearization nonlinear systems and was extended to the class of nonlinear systems in [20]. Its main idea is to introduce predefined performance bounds of the tracking errors and is able to adjust control performance indices. However, to the author's best knowledge, by far, the prescribed performance design methodology has not been applied to nonlinear strictfeedback systems with unknown functions and immeasurable states, which is important and more practical; thus, it has motivated us for this study.

In this paper, an adaptive fuzzy output-feedback control design with prescribed performance is developed for a class of uncertain SISO nonlinear stochastic systems with unmeasured states. With the help of fuzzy logic systems identifying the unknown nonlinear systems, a fuzzy adaptive observer is developed to estimate the immeasurable states. The backstepping control design technique based on predefined performance bounds is presented to design adaptive fuzzy output-feedback controller. It is shown that all the signals of the resulting closed-loop system are bounded in probability. Moreover, the tracking error converges to an adjustable neighborhood of the origin and remains within the prescribed performance bounds. Compared with the existing results, the main advantages of the proposed control scheme are as follows: (i) the restrictive assumption that all the states of the system be measured directly can be removed by designing a state observer; and (ii) by introducing predefined performance, the proposed adaptive control method can ensure that the tracking error converges to a predefined arbitrarily small residual set.

\section{System Descriptions and Preliminaries}

2.1. Nonlinear System Descriptions. Consider the following SISO strict-feedback nonlinear stochastic system:

$$
\begin{aligned}
d x_{1}= & \left(x_{2}+f_{1}\left(x_{1}\right)+d_{1}(t)\right) d t+g_{1}(x) d w \\
d x_{2}= & \left(x_{3}+f_{2}\left(\bar{x}_{2}\right)+d_{2}(t)\right) d t+g_{2}(x) d w \\
\vdots & \\
d x_{n-1}= & \left(x_{n}+f_{n-1}\left(\bar{x}_{n-1}\right)+d_{n-1}(t)\right) d t \\
& +g_{n-1}(x) d w
\end{aligned}
$$

$$
\begin{aligned}
d x_{n} & =\left(u+f_{n}\left(\bar{x}_{n}\right)+d_{n}(t)\right) d t+g_{n}(x) d w, \\
y & =x_{1},
\end{aligned}
$$

where $\bar{x}_{i}=\left[x_{1}, x_{2}, \ldots, x_{i}\right]^{T} \in R^{i}, i=1,2, \ldots, n(x=$ $\left.\underline{x}_{n}\right)$ is the state vector; $u \in R$ and $y \in R$ are the control input and system output, respectively. $f_{i}\left(\bar{x}_{i}\right)$ and $g_{i}(x) i=$ $1,2, \ldots, n$ are unknown continuous nonlinear functions, and $d_{i}(t), i=1,2, \ldots, n$ is the external disturbance. $w \in R$ is an independent standard Wiener process defined on a complete probability space with the incremental covariance $E\left\{d w \cdot d w_{j}^{T}\right\}=\sigma(t) \sigma(t)^{T} d t$.

In this paper, the states $x_{i}(i \geq 2)$ are assumed not to be available for measurement.

Our control objective is to design a stable output feedback control scheme for system (1) to ensure that all the signals are bounded in probability and that the system output $y(t)$ can track the given reference signal $y_{d}(t)$ with the given prescribed performance bounds.

Assumption 1. The external disturbances $d_{i}(t)$ are bounded; that is, $\left|d_{i}(t)\right| \leq d_{i}^{*}$ with $d_{i}^{*}$ being an unknown constant.

Assumption 2 (see [17]). Assume that functions $f_{i}(\cdot)$ satisfy the global Lipschitz condition; that is, there exist known constants $m_{i}, i=1,2, \ldots, n$ such that for all $X_{1}, X_{2} \in R^{i}$, the following inequalities hold:

$$
\left|f_{i}\left(X_{1}\right)-f_{i}\left(X_{2}\right)\right| \leq m_{i}\left\|X_{1}-X_{2}\right\|,
$$

where $\|X\|$ denotes the 2-norm of a vector $X$.

Assumption 3 (see [9]). The disturbance covariance $g^{T} \sigma \sigma^{T} g=\bar{\sigma} \bar{\sigma}^{T}$ is bounded, where $g=\left[g_{1}, \ldots, g_{n}\right]^{T}$.

2.2. Prescribed Performance. This section introduces preliminary knowledge on the prescribed performance concept reported in [20]. According to [20], the prescribed performance is achieved by ensuring that each error $z_{i}(t)$ evolves strictly within predefined decaying bounds as follows:

$$
-\delta_{i \min } \mu_{i}(t)<z_{i}(t)<\delta_{i \max } \mu_{i}(t), \quad \forall t \geq 0,
$$

where $1 \leq i \leq n, \delta_{i \min }$ and $\delta_{i \max }$ are design constants, and the performance functions $\mu_{i}(t)$ are bounded and strictly positive decreasing smooth functions with the property $\lim _{t \rightarrow \infty} \mu_{i}(t)=\mu_{i, \infty} ; \mu_{i, \infty}>0$ are a constant. In this paper, the performance functions are chosen as the exponential form $\mu_{i}(t)=\left(\mu_{i, 0}-\mu_{i, \infty}\right) e^{-n_{i} t}+\mu_{i, \infty}$, where $n_{i}, \mu_{i, 0}$, and $\mu_{i, \infty}$ are strictly positive constants, $\mu_{i, 0}>\mu_{i, \infty}$, and $\mu_{i, 0}=\mu_{i}(0)$ is selected such that $-\delta_{i \min } \mu_{i}(0)<$ $z_{i}(0)<\delta_{i \max } \mu_{i}(0)$ is satisfied. The constant $\mu_{i, \infty}$ denotes the maximum allowable size of $z_{i}(t)$ at steady state that is adjustable to an arbitrary small value reflecting the resolution of the measurement device. The decreasing rate $n_{i}$ represents a lower bound on the required speed of convergence of $z_{i}(t)$. Furthermore, the maximum overshoot of $z_{i}(t)$ is prescribed less than $\max \left\{\delta_{i \min } \mu_{i}(0), \delta_{i \max } \mu_{i}(0)\right\}$. Therefore, choosing the performance function $\mu_{i}(t)$ and the constants $\delta_{i \min }, \delta_{i \max }$ 
appropriately determines the performance bounds of the error $z_{i}(t)$.

To represent (3) by an equality form, we employ an error transformation as

$$
z_{i}=\mu_{i}(t) \Phi_{i}\left(\zeta_{i}(t)\right), \quad \forall t \geq 0,
$$

where $\Phi_{i}\left(\zeta_{i}\right)=\left(\delta_{i \max } e^{\zeta_{i}}-\delta_{i \min } e^{-\zeta_{i}}\right) /\left(e^{\zeta_{i}}+e^{-\zeta_{i}}\right)$

Since the function $\Phi_{i}\left(\zeta_{i}\right)$ is strictly monotonic increasing, its inverse function can be expressed as

$$
\begin{gathered}
\zeta_{i}(t)=\Phi^{-1}\left(\frac{z_{i}(t)}{\mu_{i}(t)}\right)=\frac{1}{2} \ln \frac{\Phi_{i}-\delta_{i \min }}{\delta_{i \max }-\Phi_{i}}, \\
\dot{\zeta}_{i}(t)=p_{i}\left(\dot{z}_{i}-\frac{\dot{\mu}_{i} z_{i}}{\mu_{i}}\right)
\end{gathered}
$$

with $p_{i}=\left(1 / 2 \mu_{i}\right)\left[\left(1 /\left(\Phi_{i}+\delta_{i \min }\right)\right)-\left(1 /\left(\Phi_{i}-\delta_{i \max }\right)\right)\right]$.

For the output-feedback control design of the nonlinear system, we design the following state transformation:

$$
z_{i}(t)=\zeta_{i}(t)-\frac{1}{2} \ln \frac{\delta_{i \min }}{\delta_{i \max }} .
$$

And the transformation state dynamics is

$$
\dot{z}_{i}(t)=p_{i}\left(\dot{z}_{i}-\frac{\dot{\mu}_{i} z_{i}}{\mu_{i}}\right) .
$$

2.3. Fuzzy Logic Systems. A fuzzy logic system (FLS) consists of four parts: the knowledge base, the fuzzifier, the fuzzy inference engine working on fuzzy rules, and the defuzzifier. The knowledge base for FLS comprises a collection of fuzzy IF-THEN rules of the following form:

$$
\begin{aligned}
& R^{l}: \text { If } x_{1} \text { is } F_{1}^{l}, x_{2} \text { is } F_{2}^{l}, \ldots, x_{n} \text { is } F_{n}^{l}, \\
& \text { Then } y \text { is } G^{l}, \quad l=1,2, \ldots, N,
\end{aligned}
$$

where $x=\left(x_{1}, \ldots, x_{n}\right)^{T}$ and $y$ are the FLS input and output, respectively. Fuzzy sets $F_{i}^{l}$ and $G^{l}$ are associated with the fuzzy functions $\mu_{F_{i}^{l}}\left(x_{i}\right)$ and $\mu_{G^{l}}(y)$, respectively. $N$ is the rule number of IF-THEN.

Through singleton function, center average defuzzification, and product inference [21], the FLS can be expressed as

$$
y(x)=\frac{\sum_{l=1}^{N} \bar{y}_{l} \prod_{i=1}^{n} \mu_{F_{i}^{l}}\left(x_{i}\right)}{\sum_{l=1}^{N}\left[\prod_{i=1}^{n} \mu_{F_{i}^{l}}\left(x_{i}\right)\right]},
$$

where $\bar{y}_{l}=\max _{y \in R} \mu_{G^{l}}(y)$.

Define the fuzzy basis functions as

$$
\varphi_{l}=\frac{\prod_{i=1}^{n} \mu_{F_{i}^{l}}\left(x_{i}\right)}{\sum_{l=1}^{N}\left(\prod_{i=1}^{n} \mu_{F_{i}^{l}}\left(x_{i}\right)\right)} .
$$

Denoting $\theta^{T}=\left[\bar{y}_{1}, \bar{y}_{2}, \ldots, \bar{y}_{N}\right]=\left[\theta_{1}, \theta_{2}, \ldots, \theta_{N}\right]$ and $\varphi(x)=$ $\left[\varphi_{1}(x), \ldots, \varphi_{N}(x)\right]^{T}$, then FLS (9) can be rewritten as

$$
y(x)=\theta^{T} \varphi(x) .
$$

Lemma 4 (see [21]). Let $f(x)$ be a continuous function defined on a compact set $\Omega$. Then for any constant $\varepsilon>0$, there exists a FLS (11) such as

$$
\sup _{x \in \Omega}\left|f(x)-\theta^{T} \varphi(x)\right| \leq \varepsilon .
$$

\section{Fuzzy State Observer Design}

Since the states $x_{2}, \ldots, x_{n}$ in system (1) are not available for measurement, a state observer is to be established to estimate them in this section.

Rewrite (1) in the following form:

$$
\begin{aligned}
& d \bar{x}_{n} \\
& =\left(A \bar{x}_{n}+K y+\sum_{i=1}^{n} B_{i}\left[f_{i}\left(\bar{x}_{i}\right)+d_{i}(t)\right]+B u\right) d t \\
& \quad+g(x) d w \\
& =\left(A \bar{x}_{n}+K y+\sum_{i=1}^{n} B_{i}\left[f_{i}\left(\hat{\bar{x}}_{i}\right)+\Delta f_{i}+d_{i}(t)\right]+B u\right) d t \\
& \quad+g(x) d w,
\end{aligned}
$$

where $\widehat{\bar{x}}_{i}=\left(\widehat{x}_{1}, \widehat{x}_{2}, \ldots, \widehat{x}_{i}\right)^{T}$ is the estimate of $\bar{x}_{i}=$

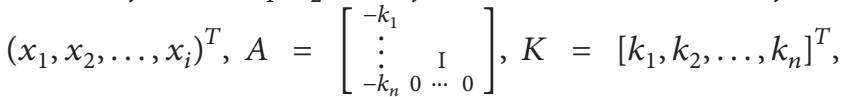
$B_{i}=[0 \cdots 1 \cdots 0]^{T}, B=[0 \cdots 0 \cdots 1]^{T}, \Delta f_{i}=f_{i}\left(\bar{x}_{i}\right)-f_{i}\left(\hat{\bar{x}}_{i}\right)$, $g(x)=\left[g_{1}(x), \ldots, g_{n}(x)\right]^{T}$.

The vector $K$ is chosen such that $A$ is a Hurwitz matrix. Thus, given a positive definite matrix $Q=Q^{T}>0$, there exists a positive definite matrix $P=P^{T}>0$ satisfying

$$
A^{T} P+P A=-2 Q \text {. }
$$

By Lemma 4 , we can assume that nonlinear terms $f_{i}\left(\hat{\bar{x}}_{i}\right), i=$ $1,2, \ldots, n$ in (13) can be approximated by the following FLSs:

$$
\widehat{f}_{i}\left(\hat{\bar{x}}_{i} \theta_{i}\right)=\theta_{i}^{T} \varphi_{i}\left(\hat{\bar{x}}_{i}\right) \text {. }
$$

Define the optimal parameter vectors $\theta_{i}^{*}$ as

$$
\theta_{i}^{*}=\underset{\theta_{i} \in \Omega_{i}}{\arg \min }\left[\sup _{\hat{\bar{x}}_{i} \in U_{i}}\left|\widehat{f}_{i}\left(\hat{\bar{x}}_{i} \mid \theta_{i}\right)-f_{i}\left(\hat{\bar{x}}_{i}\right)\right|\right],
$$

where $\Omega_{i}$ and $U_{i}$ are bounded compact sets for $\theta_{i}$ and $\widehat{\bar{x}}_{i}$, respectively. Also, the fuzzy minimum approximation error $\varepsilon_{i}$ is defined as

$$
\varepsilon_{i}=f_{i}\left(\hat{\bar{x}}_{i}\right)-\widehat{f}_{i}\left(\hat{\bar{x}}_{i} \mid \theta_{i}^{*}\right),
$$

where $\varepsilon_{i}$ satisfies $\left|\varepsilon_{i}\right| \leq \varepsilon_{i}^{*}$, with $\varepsilon_{i}^{*}$ being a positive constant.

The state observer for (13) is designed as

$$
\begin{gathered}
\dot{\overline{\bar{x}}}_{n}=A \widehat{\bar{x}}_{n}+K y+\sum_{i=1}^{n} B_{i}\left[\widehat{f}_{i}\left(\hat{\bar{x}}_{i} \mid \theta_{i}\right)\right]+B u, \\
\hat{y}=C \widehat{\bar{x}}_{n},
\end{gathered}
$$

where $C=[1 \cdots 0 \cdots 0]$. 


\section{Adaptive Controller Design}

In this section, an adaptive fuzzy output-feedback control scheme will be developed by using the above fuzzy state observer and the backstepping technique, and the stability of the closed-loop system will be given.

The controller design consists of step $n$; each step is based on the following change of coordinates:

$$
z_{1}=y-y_{d}, \quad z_{i}=\widehat{x}_{i}-\alpha_{i-1}, \quad(i=2, \ldots, n)
$$

where $\alpha_{i-1}$ is referred to as the intermediate control function, which will be designed later.

Step 1. From (1), (7), and (19), according to Itô's differentiation rule, we can obtain

$$
\begin{gathered}
d z_{1}=p_{1}\left(x_{2}+f_{1}\left(x_{1}\right)+d_{1}-\dot{y}_{d}-\frac{\dot{\mu}_{1} z_{1}}{\mu_{1}}\right) d t \\
+p_{1} g_{1}(x) d w \\
=p_{1}\left(z_{2}+\alpha_{1}+e_{2}+\theta_{1}^{T} \varphi_{1}\left(\widehat{x}_{1}\right)\right. \\
+\widetilde{\theta}_{1}^{T} \varphi_{1}\left(\widehat{x}_{1}\right)+\varepsilon_{1}+d_{1}-\dot{y}_{d} \\
\left.+\Delta f_{1}-\frac{\dot{\mu}_{1} z_{1}}{\mu_{1}}\right) d t+p_{1} g_{1}(x) d w .
\end{gathered}
$$

Choose the intermediate control function $\alpha_{1}$ and the adaptation law for $\theta_{1}$ as follows:

$$
\begin{gathered}
\alpha_{1}=-c_{1} z_{1} p_{1}-\frac{9}{4} z_{1} p_{1}^{1 / 3}-\frac{3}{4} z_{1} p_{1}^{3}-z_{1}^{3} p_{1} \\
-\theta_{1}^{T} \varphi_{1}\left(\widehat{x}_{1}\right)+\dot{y}_{d}+\frac{\dot{\mu}_{1} z_{1}}{\mu_{1}}, \\
\dot{\theta}_{1}=\eta_{1} z_{1}^{3} p_{1} \varphi_{1}\left(\widehat{x}_{1}\right)-\sigma_{1} \theta_{1},
\end{gathered}
$$

where $c_{1}>0, \sigma_{1}>0$ and $\eta_{1}>0$ are design parameters and $\theta_{1}$ is the estimate of $\theta_{1}^{*}$.

Step $i(2 \leq i \leq n-1)$. Similar to Step 1, we have

$$
\begin{aligned}
d z_{i}=\left[p _ { i } \left(z_{i+1}+\alpha_{i}+k_{i} e_{1}+\theta_{i}^{T} \varphi\left(\hat{\bar{x}}_{i}\right)\right.\right. \\
\quad-\sum_{l=1}^{i-1} \frac{\partial \alpha_{l-1}}{\partial \hat{x}_{l}} \dot{\hat{x}}_{l}-\sum_{l=1}^{i-1} \frac{\partial \alpha_{l-1}}{\partial \theta_{l}} \dot{\theta}_{l} \\
\left.\left.\quad-\sum_{l=1}^{i-1} \frac{\partial \alpha_{l-1}}{\partial y_{d}^{(l-1)}} y_{d}^{l}-\frac{\partial \alpha_{1}}{\partial y} \dot{y}-\frac{\dot{\mu}_{i} z_{i}}{\mu_{i}}\right)\right] d t \\
-p_{i} \frac{\partial \alpha_{i-1}}{\partial y} g_{1}(x) d w
\end{aligned}
$$

$$
\begin{aligned}
& =\left[p _ { i } \left(z_{i+1}+\alpha_{i}+k_{i} e_{1}+\theta_{i}^{T} \varphi\left(\hat{\bar{x}}_{i}\right)\right.\right. \\
& +\widetilde{\theta}_{i}^{T} \varphi\left(\hat{\bar{x}}_{i}\right)-\widetilde{\theta}_{i}^{T} \varphi\left(\hat{\bar{x}}_{i}\right)-\frac{\partial \alpha_{1}}{\partial y} \\
& \quad \times\left[\widetilde{\theta}_{1}^{T} \varphi_{1}\left(\widehat{x}_{1}\right)+e_{2}+\varepsilon_{1}+\Delta f_{1}+d_{1}\right] \\
& \left.\left.\quad-H_{i}-\frac{\dot{\mu}_{i} z_{i}}{\mu_{i}}\right)\right] d t \\
& -p_{i} \frac{\partial \alpha_{i-1}}{\partial y} g_{1}(x) d w
\end{aligned}
$$

where

$$
\begin{aligned}
H_{i}= & -\sum_{l=1}^{i-1} \frac{\partial \alpha_{l-1}}{\partial \widehat{x}_{l}}\left[\widehat{x}_{l+1}+\theta_{l}^{T} \varphi_{l}\left(\hat{\bar{x}}_{l}\right)+k_{l} e_{1}\right] \\
& -\frac{\partial \alpha_{1}}{\partial \theta_{1}} \dot{\theta}_{1}-\frac{\partial \alpha_{1}}{\partial y_{d}^{(l-1)}} \dot{y}_{d}-\frac{\partial \alpha_{1}}{\partial y}\left[\widehat{x}_{2}+\theta_{1}^{T} \varphi_{1}\left(\widehat{x}_{1}\right)\right] .
\end{aligned}
$$

Choose intermediate control function $\alpha_{i}$ and adaptation law $\theta_{i}$ as

$$
\begin{gathered}
\alpha_{i}=-c_{i} z_{i} p_{i}-k_{i} e_{1}-\bar{H}_{i}-\theta_{i}^{T} \varphi_{i}\left(\hat{\bar{x}}_{i}\right) \\
-\frac{3}{4} z_{i} p_{i}^{1 / 3}-\frac{1}{4} z_{i}+\frac{\dot{\mu}_{i} z_{i}}{\mu_{i}}, \\
\dot{\theta}_{i}=\eta_{i} z_{i}^{3} p_{i} \varphi_{i}\left(\hat{\bar{x}}_{i}\right)-\sigma_{i} \theta_{i},
\end{gathered}
$$

where $c_{i}>0, \sigma_{i}>0$ and $\eta_{i}>0$ are design parameters and $\theta_{i}$ is the estimate of $\theta_{i}^{*}$, and

$$
\begin{aligned}
\bar{H}_{i}= & H_{i}+\left(\frac{\partial \alpha_{i-1}}{\partial x_{1}}\right)^{2} z_{i}^{3} p_{i}+\frac{3}{4} z_{i} p_{i}^{3}\left(\frac{\partial \alpha_{i-1}}{\partial y}\right)^{4} \\
& +\frac{1}{2} z_{i}^{3} p_{i}+\frac{1}{4} z_{i}^{3} p_{i}\left(\frac{\partial^{2} \alpha_{i-1}}{\partial y^{2}}\right)^{2} .
\end{aligned}
$$

Step $n$. In the final design step, the actual control input $u$ will be designed. Similar to Step $i$ we have

$$
\begin{aligned}
d z_{n}= & p_{n}\left(u+k_{n} e_{1}+\theta_{n}^{T} \varphi_{n}\left(\hat{\bar{x}}_{n}\right)-\dot{\alpha}_{n-1}-\frac{\dot{\mu}_{n} z_{n}(t)}{\mu_{n}}\right) d t \\
& -p_{n} \frac{\partial \alpha_{n-1}}{\partial y} g_{1}(x) d w .
\end{aligned}
$$

The controller $u$ and adaptation law $\theta_{n}$ are chosen as

$$
\begin{gathered}
u=-c_{n} z_{n} p_{n}-k_{n} e_{1}-\theta_{n}^{T} \varphi_{n}\left(\hat{\bar{x}}_{n}\right)-\bar{H}_{n}-\frac{1}{4} z_{n}+\frac{\dot{\mu}_{n} z_{n}}{\mu_{n}}, \\
\dot{\theta}_{n}=\eta_{n} z_{n}^{3} p_{n} \varphi_{n}\left(\hat{\bar{x}}_{n}\right)-\sigma_{n} \theta_{n}
\end{gathered}
$$

where $c_{n}>0, \sigma_{n}>0$ and $\eta_{i}>0$ are design parameters and $\theta_{n}$ is the estimate of $\theta_{n}^{*}$. 


\section{Stability Analysis}

Consider the total Lyapunov candidate functions $V$ as the sum of local Lyapunov candidate functions $V_{0}$ and $V_{i}$, namely, $V=V_{0}+V_{i}$, with $V_{0}=(1 / 2) e^{T} P e$, and $V_{i}=\sum_{i=1}^{n}\left((1 / 4) z_{i}^{4}+\right.$ $\left.\left(1 / 2 \eta_{i}\right) \widetilde{\theta}_{i}^{T} \widetilde{\theta}_{i}\right)$, where $e=\bar{x}-\widehat{\bar{x}}$ is the observer error vector, $\eta_{i}$ is positive design constant, and $\widetilde{\theta}_{i}=\theta_{i}^{*}-\theta_{i}$.

Theorem 5. For the stochastic nonlinear system (1), if Assumptions 1-3 are satisfied, the controller (29) with the state observer (18), together with the intermediate control functions (21) and (25), and adaptation laws (22), (26), and (30) can guarantee that all signals in the closed-loop system are semiglobally uniformly ultimately bounded in probability, and the tracking error remains in a neighborhood of the origin within the prescribed performance bounds for all $t \geq 0$.

Proof. The infinitesimal generator of $V$ is

$$
\ell V=\ell V_{0}+\ell V_{i}
$$

From (13) and (18), we have the observer error equation

$$
\begin{array}{r}
d e=\left(A e+\sum_{i=1}^{n} B_{i}\left[f_{i}\left(\hat{\bar{x}}_{i}\right)-\widehat{f}_{i}\left(\hat{\bar{x}}_{i} \mid \theta_{i}\right)\right.\right. \\
\left.\left.+\Delta f_{i}+d_{i}\right]\right) d t+g(x) d w \\
=\left(A e+\sum_{i=1}^{n} B_{i}\left[\varepsilon_{i}+\widetilde{\theta}_{i}^{T} \varphi_{i}\left(\hat{\bar{x}}_{i}\right)\right.\right. \\
\left.\left.+\Delta f_{i}+d_{i}\right]\right) d t+g(x) d w \\
=\left(\begin{array}{c}
A e+\varepsilon+d+\Delta f \\
\left.+\sum_{i=1}^{n} B_{i} \widetilde{\theta}_{i}^{T} \varphi_{i}\left(\hat{\bar{x}}_{i}\right)\right) d t+g(x) d w
\end{array}\right.
\end{array}
$$

where $\Delta f=\left[\Delta f_{1}, \ldots, \Delta f_{n}\right]^{T}, \varepsilon=\left[\varepsilon_{1}, \ldots, \varepsilon_{n}\right]^{T}, d=$ $\left[d_{1}, \ldots, d_{n}\right]^{T}, \widetilde{\theta}_{i}=\theta_{i}^{*}-\theta_{i}$.

The infinitesimal generator of $V_{0}$ along with (32) is

$$
\begin{aligned}
\ell V_{0} \leq & -\lambda_{\min }(Q)\|e\|^{2}+e^{T} P(\varepsilon+d+\Delta f) \\
& +\sum_{i=1}^{n} e^{T} P B_{i} \widetilde{\theta}_{i}^{T} \varphi_{i}\left(\hat{\bar{x}}_{i}\right)+\operatorname{Tr}\left[\sigma g^{T} P g \sigma^{T}\right] .
\end{aligned}
$$

By Young's inequality, Assumptions 1-3, we have

$$
\begin{aligned}
e^{T} P(d+\varepsilon+\Delta f) \leq & \frac{3}{2}\|e\|^{2}+\frac{1}{2}\|P\|^{2}\left\|\mathcal{E}^{*}\right\|^{2} \\
& +\frac{1}{2}\|P\|^{2}\left\|d^{*}\right\|^{2}+\frac{1}{2}\|P\|^{2}\|\Delta f\|^{2}
\end{aligned}
$$

$$
\begin{aligned}
\leq & \left(\frac{3}{2}+\frac{1}{2}\|P\|^{2} \sum_{i=1}^{n} m_{i}^{2}\right)\|e\|^{2} \\
& +\frac{1}{2}\|P\|^{2}\left\|\varepsilon^{*}\right\|^{2}+\frac{1}{2}\|P\|^{2}\left\|d^{*}\right\|^{2}, \\
\operatorname{Tr}\left[\sigma g^{T} P g \sigma^{T}\right] & \leq \frac{1}{2}\|P\|^{2}+\frac{1}{2}\left|\bar{\sigma} \bar{\sigma}^{T}\right|^{2},
\end{aligned}
$$

where $\varepsilon^{*}=\left[\varepsilon_{1}^{*}, \ldots, \varepsilon_{n}^{*}\right]^{T}, d^{*}=\left[d_{1}^{*}, \ldots, d_{n}^{*}\right]^{T}$.

Note that $\varphi_{i}^{T}\left(\widehat{\bar{x}}_{i}\right) \varphi_{i}\left(\widehat{\bar{x}}_{i}\right) \leq 1$; by Young's inequality, we have

$$
\begin{aligned}
e^{T} P \sum_{i=1}^{n} B_{i} \widetilde{\theta}_{i}^{T} \varphi_{i}\left(\hat{\bar{x}}_{i}\right) & \leq \frac{1}{4} e^{T} P P^{T} e+\sum_{i=1}^{n} \widetilde{\theta}_{i}^{T} \varphi_{i}\left(\widehat{\bar{x}}_{i}\right) \varphi_{i}^{T}\left(\widehat{\bar{x}}_{i}\right) \widetilde{\theta}_{i} \\
& \leq \frac{1}{4} \lambda_{\max }^{2}(P)\|e\|^{2}+\sum_{i=1}^{n} \widetilde{\theta}_{i}^{T} \widetilde{\theta}_{i},
\end{aligned}
$$

where $\lambda_{\text {max }}(P)$ is the largest eigenvalue of $P$.

Substituting (34)-(35) into (33) gives

$$
\dot{V}_{0} \leq-q_{0}\|e\|^{2}+\sum_{i=1}^{n} \widetilde{\theta}_{i}^{T} \widetilde{\theta}_{i}+\lambda_{0},
$$

where $q_{0}=\lambda_{\min }(Q)-\left((3 / 2)+(1 / 2)\|P\|^{2} \sum_{i=1}^{n} m_{i}^{2}+\right.$ $\left.(1 / 4) \lambda_{\text {max }}^{2}(P)\right), \lambda_{0}=(1 / 2)\|P\|^{2}\left\|\varepsilon^{*}\right\|^{2}+(1 / 2)\|P\|^{2}\left\|d^{*}\right\|^{2}+$ $(1 / 2)\|P\|^{2}+(1 / 2)\left|\bar{\sigma} \bar{\sigma}^{T}\right|^{2}$, and $\lambda_{\min }(Q)$ is the minimal eigenvalue of $Q$.

From (19), (20), (23), and (28) we have

$$
\begin{aligned}
z_{1}^{3} \dot{z}_{1}= & z_{1}^{3} p_{1}\left(x_{2}+f_{1}\left(x_{1}\right)+d_{1}-\dot{y}_{d}-\frac{\dot{\mu}_{1} z_{1}}{\mu_{1}}\right) \\
& +\frac{3}{2} z_{1}^{2} p_{1}^{2} g_{1}^{T} \sigma \sigma^{T} g_{1} \\
= & z_{1}^{3} p_{1}\left(z_{2}+\alpha_{1}+e_{2}+\theta_{1}^{T} \varphi_{1}\left(\widehat{x}_{1}\right)+\widetilde{\theta}_{1}^{T} \varphi_{1}\left(\widehat{x}_{1}\right)\right. \\
& \left.+\varepsilon_{1}+d_{1}-\dot{y}_{d}+\Delta f_{1}-\frac{\dot{\mu}_{1} z_{1}}{\mu_{1}}\right) \\
& +\frac{3}{2} z_{1}^{2} p_{1}^{2} g_{1}^{T} \sigma \sigma^{T} g_{1},
\end{aligned}
$$

$$
\begin{aligned}
z_{i}^{3} \dot{z}_{i}=z_{i}^{3} p_{i}( & z_{i+1}+\alpha_{i}+k_{i} e_{1}-\frac{\partial \alpha_{1}}{\partial y} \\
& \times\left[\widetilde{\theta}_{1}^{T} \varphi_{1}\left(\widehat{x}_{1}\right)+e_{2}+\Delta f_{1}+d_{1}+\varepsilon_{1}\right] \\
& +\theta_{i}^{T} \varphi_{i}\left(\hat{\bar{x}}_{i}\right)+\widetilde{\theta}_{i}^{T} \varphi_{i}\left(\hat{\bar{x}}_{i}\right)-\widetilde{\theta}_{i}^{T} \varphi_{i}\left(\hat{\bar{x}}_{i}\right)-H_{i} \\
& \left.-\frac{1}{2} \frac{\partial^{2} \alpha_{i-1}}{\partial y^{2}} g_{1}^{T} \sigma \sigma^{T} g_{1}-\frac{\dot{\mu}_{i} z_{i}}{\mu_{i}}\right) \\
+ & \frac{3}{2} p_{i}^{2} z_{i}^{2}\left(\frac{\partial \alpha_{i-1}}{\partial y}\right)^{2} g_{1}^{T} \sigma \sigma^{T} g_{1}
\end{aligned}
$$




$$
\begin{aligned}
z_{n}^{3} \dot{z}_{n}= & z_{n}^{3} p_{n}\left(\theta_{n}^{T} \varphi_{n}\left(\hat{\bar{x}}_{n}\right)+u+\frac{1}{4} z_{n}+k_{n} e_{1}-\bar{H}_{n}-\frac{\dot{\mu}_{n} z_{n}(t)}{\mu_{n}}\right) \\
& +\widetilde{\theta}_{n}^{T} \varphi_{n}\left(\hat{\bar{x}}_{n}\right)-\widetilde{\theta}_{n}^{T} \varphi_{n}\left(\hat{\bar{x}}_{n}\right)+\frac{3}{2} p_{n}^{2} z_{n}^{2}\left(\frac{\partial \alpha_{n-1}}{\partial y}\right)^{2} g_{1}^{T} \sigma \sigma^{T} g_{1} .
\end{aligned}
$$

By Young's inequality and Assumptions 1-3, we have

$$
\begin{aligned}
& z_{1}^{3} p_{1} z_{2}+z_{1}^{3} p_{1} e_{2}+z_{1}^{3} p_{1} \varepsilon_{1}+z_{1}^{3} p_{1} d_{1}+z_{1}^{3} p_{1} \Delta f_{1} \\
& \leq \frac{3}{4} z_{1}^{4} p_{1}^{4 / 3}+\frac{1}{4} z_{2}^{4}+\frac{1}{2} z_{1}^{6} p_{1}^{2}+\frac{1}{2}\|e\|^{2}+\frac{3}{4} z_{1}^{4} p_{1}^{4 / 3} \\
& +\frac{1}{4} \varepsilon_{1}^{* 4}+\frac{3}{4} z_{1}^{4} p_{1}^{4 / 3}+\frac{1}{4} d_{1}^{* 4}+\frac{1}{2} z_{1}^{6} p_{1}^{2}+\frac{1}{2} \Delta f_{1}^{2} \\
& \leq \frac{9}{4} z_{1}^{4} p_{1}^{4 / 3}+z_{1}^{6} p_{1}^{2}+\frac{1}{4} z_{2}^{4}+\frac{1}{4} \varepsilon_{1}^{* 4}+\frac{1}{4} d_{1}^{* 4} \\
& +\frac{1}{2}\|e\|^{2}+\frac{1}{2}\|e\|^{2} m_{1}^{2}, \\
& \frac{3}{2} z_{1}^{2} p_{1}^{2} g_{1}^{T} \sigma \sigma^{T} g_{1} \leq \frac{3}{4} z_{1}^{4} p_{1}^{4}+\frac{3}{4}\left|\bar{\sigma} \bar{\sigma}^{T}\right|^{2}, \\
& z_{i}^{3} p_{i} z_{i+1}-z_{i}^{3} p_{i} \widetilde{\theta}_{i}^{T} \varphi_{i}\left(\widehat{\bar{x}}_{i}\right) \\
& \leq \frac{3}{4} z_{i}^{4} p_{i}^{4 / 3}+\frac{1}{4} z_{i+1}^{4}+\frac{1}{2} z_{i}^{6} p_{i}^{2}+\frac{1}{2} \widetilde{\theta}_{i}^{T} \widetilde{\theta}_{i}, \\
& -z_{i}^{3} p_{i} \frac{\partial \alpha_{i-1}}{\partial x_{1}} e_{2} \leq \frac{1}{2}\|e\|^{2}+\frac{1}{2}\left(\frac{\partial \alpha_{i-1}}{\partial x_{1}}\right)^{2} z_{i}^{6} p_{i}^{2}, \\
& -z_{i}^{3} p_{i} \frac{\partial \alpha_{i-1}}{\partial x_{1}} \widetilde{\theta}_{1}^{T} \varphi_{1}\left(\widehat{x}_{1}\right) \leq \frac{1}{2} \widetilde{\theta}_{1}^{T} \widetilde{\theta}_{1}+\frac{1}{2}\left(\frac{\partial \alpha_{i-1}}{\partial x_{1}}\right)^{2} z_{i}^{6} p_{i}^{2}, \\
& -z_{i}^{3} p_{i} \frac{\partial \alpha_{i-1}}{\partial x_{1}}\left[\varepsilon_{1}+\Delta f_{1}+d_{1}\right] \\
& \leq \frac{3}{2}\left(\frac{\partial \alpha_{i-1}}{\partial x_{1}}\right)^{2} z_{i}^{6} p_{i}^{2}+\frac{1}{2} \varepsilon_{1}^{* 2}+\frac{1}{2} d_{1}^{* 2}+\frac{m_{1}^{2}}{2}\|e\|^{2}, \\
& -\frac{1}{2} z_{i}^{3} p_{i} \frac{\partial^{2} \alpha_{i-1}}{\partial y^{2}} g_{1}^{T} \sigma \sigma^{T} g_{1}+\frac{3}{2} p_{i}^{2} z_{i}^{2}\left(\frac{\partial \alpha_{i-1}}{\partial y}\right)^{2} g_{1}^{T} \sigma \sigma^{T} g_{1} \\
& \leq \frac{1}{4} z_{i}^{6} p_{i}^{2}\left(\frac{\partial^{2} \alpha_{i-1}}{\partial y^{2}}\right)^{2}+\frac{1}{4}|\bar{\sigma} \bar{\sigma}|^{2}+\frac{3}{4} p_{i}^{4} z_{i}^{4}\left(\frac{\partial \alpha_{i-1}}{\partial y}\right)^{4} \\
& +\frac{3}{4}\left|\bar{\sigma} \bar{\sigma}^{T}\right|^{2} \text {. }
\end{aligned}
$$

From (21)-(22), (25)-(26), (29)-(30), and (41)-(44), we have

$$
\begin{aligned}
\ell V \leq & -q_{n}\|e\|^{2}-\sum_{i=1}^{n} c_{i} z_{i}^{4} p_{i}^{2}+\sum_{i=1}^{n} \frac{\sigma_{i}}{\eta_{i}} \widetilde{\theta}_{i}^{T} \theta_{i} \\
& +2 \sum_{i=1}^{n} \widetilde{\theta}_{i}^{T} \widetilde{\theta}_{i}+\frac{n-1}{2} \widetilde{\theta}_{1}^{T} \widetilde{\theta}_{1}+\lambda_{n}
\end{aligned}
$$

where $q_{n}=q_{0}-(n / 2)-\left(n m_{1}^{2} / 2\right), \lambda_{n}=\lambda_{0}+(n / 2) \varepsilon_{1}^{2}+(n / 2) d_{1}^{* 2}+$ $n|\bar{\sigma} \bar{\sigma}|^{2}$.
Note that

$$
\sum_{i=1}^{n} \frac{\sigma_{i}}{\eta_{i}} \widetilde{\theta}_{i}^{T} \theta_{i} \leq-\frac{1}{2} \sum_{i=1}^{n} \frac{\sigma_{i}}{\eta_{i}} \widetilde{\theta}_{i}^{T} \widetilde{\theta}_{i}+\frac{1}{2} \sum_{i=1}^{n} \frac{\sigma_{i}}{\eta_{i}} \theta_{i}^{* T} \theta_{i}^{*} .
$$

Substituting the above inequality into (52) gives

$$
\begin{aligned}
\ell V \leq & -q_{n}\|e\|^{2}-\sum_{i=1}^{n} c_{i} z_{i}^{4} p_{i}^{2}-\sum_{i=2}^{n}\left(\frac{\sigma_{i}}{2 \eta_{i}}-2\right) \widetilde{\theta}_{i}^{T} \widetilde{\theta}_{i} \\
& -\left(\frac{\sigma_{1}}{2 \eta_{1}}-\frac{n+3}{2}\right) \widetilde{\theta}_{1}^{T} \widetilde{\theta}_{1}+\lambda,
\end{aligned}
$$

where $D=\sum_{i=1}^{n}\left(\sigma_{i} / 2 \eta_{i}\right) \theta_{i}^{* T} \theta_{i}^{*}+\lambda_{n}$. Let $q_{n}>0, c_{i}>0$, $\left(\sigma_{i} / 2 \eta_{i}\right)>1$, and define

$$
\begin{aligned}
C=\min \left\{\frac{2 q_{n}}{\lambda_{\min }(P)}, 4 c_{i} p_{i}^{2},(i=1, \ldots, n),\right. \\
\\
\left.\sigma_{1}-(n+3) \eta_{1}, 2\left(\sigma_{i}-4 \eta_{i}\right),(i=2, \ldots, n)\right\} .
\end{aligned}
$$

Then (47) can be written as

$$
\ell V \leq-C V+D
$$

Multiplying $V$ by $e^{C t}$ and by Itô formula leads to

$$
d\left(e^{C t} V\right)=e^{C t}(C V+\ell V) d t+e^{C t} \Omega_{1} d w
$$

where $\Omega_{1}=\left(\partial V / \partial z_{1}\right) g_{1}(x)-\sum_{i=2}^{n}\left(\partial V / \partial z_{i}\right)\left(\partial \alpha_{i-1} / \partial y\right) g_{1}(x)+$ $(\partial V / \partial e) g(x)$.

From (49) and (50), we have

$$
d\left(e^{C t} V\right) \leq e^{C t} D d t+e^{C t} \Omega_{1} d w
$$

Integrating (51) over $[0, T]$, we get

$$
V(T) \leq e^{C T} V(0)+\frac{D}{C}+e^{C T} \int_{0}^{T} e^{C s} \Omega_{1} d w(s) .
$$

Taking expectation on (52), it follows that

$$
E[V(T)] \leq E V(0) e^{-C T}+\frac{D}{C},
$$

where $E(\cdot)$ is probability expectation.

The above inequality means that $E[V(T)]$ is bounded by $D / C$ in mean square. Thus, according to [12-18], it is concluded that all the signals of the closed-loop system are SGUUB in the sense of the four-moment. Moreover, it follows that the tracking errors and virtual tracking errors remain within the prescribed performance bounds for all time $t \geq$ 0 .

\section{Simulation Study}

In this section, a simulation example is provided to evaluate the control performance of the proposed adaptive outputfeedback control method. 


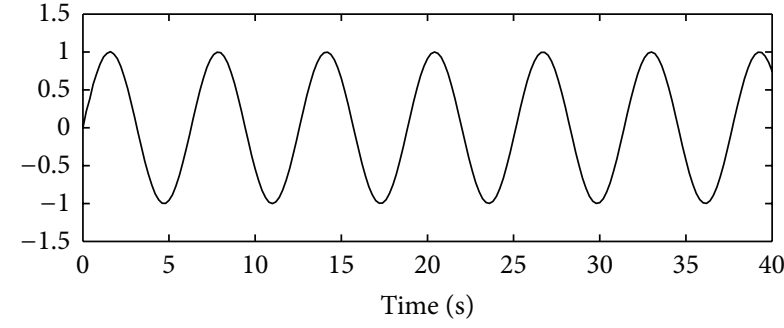

(a)

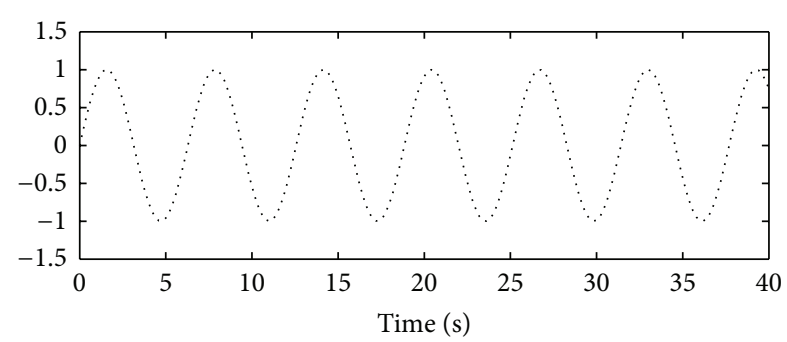

(b)

Figure 1: The curves of $y$ (solid line) and $y_{d}$ (dot line). form:

Consider a stochastic system governed by the following

$$
\begin{aligned}
d x_{1} & =\left[x_{2}+f_{1}\left(x_{1}\right)\right] d t+g_{1}(x) d w, \\
d x_{2} & =\left[f_{2}\left(x_{1}, x_{2}\right)\right] d t+u+g_{2}(x) d w, \\
y & =x_{1},
\end{aligned}
$$

where $f_{1}\left(x_{1}\right)=\sin \left(x_{1}^{2}\right), f_{2}\left(x_{1}, x_{2}\right)=x_{1} \sin \left(x_{2}^{2}\right)-x_{1} e^{0.5 x_{1}}$, $g_{1}(x)=\sin \left(x_{1}\right) /\left(1+0.5 \cos \left(x_{2}\right)\right), g_{2}(x)=x_{1} x_{2} /\left(1+\left(x_{1} x_{2}\right)^{2}\right)$. $\dot{w}(t)$ is assumed to be a Gaussian white noise with zero mean and variance 1.0. The tracking reference signal is chosen as $y_{d}(t)=\sin (t)$.

Choose fuzzy membership functions as

$$
\mu_{F_{i}^{l}}\left(\widehat{x}_{i}\right)=\exp \left[-\frac{\left(\widehat{x}_{i}-3+l\right)^{2}}{16}\right], \quad l=1,2,3,4,5 .
$$

Construct the FLSs $\widehat{f}_{i}\left(\hat{\bar{x}}_{i} \mid \theta_{i}\right)=\theta_{i}^{T} \varphi_{i}\left(\hat{\bar{x}}_{i}\right)$ to appreciate the unknown nonlinear functions $f_{i}(\cdot), i=1,2$.

Choose the design parameters and performance functions as $k_{1}=0.8, k_{2}=10, c_{1}=0.01, c_{2}=1, \eta_{1}=\eta_{2}=0.01$, $\mu_{1,0}=2, \mu_{1, \infty}=0.5, n_{1}=0.5, \sigma_{1}=\sigma_{2}=0.01, \delta_{1 \min }=0.01$, $\delta_{1 \max }=0.02$, and $\mu_{1}(t)=1.5 e^{-0.5 t}+0.5$.

The initial conditions are chosen as follows: $x_{1}(0)=0$, $x_{2}(0)=0.1, \widehat{x}_{1}(0)=0, \widehat{x}_{2}(0)=-0.1, \theta_{1}^{T}(0)=[0,0,-0.1,0,0]$, and $\theta_{2}^{T}(0)=[0,0,0,-0.1,0]$.

Applying the control method in this paper to control (54), the simulation results are shown by Figures 1-4, where Figure 1 expresses the curves of the output $y$ and tracking signal $y_{d}$; Figure 2 expresses the curves of the observer error $e_{1}$ and $e_{2}$; Figure 3 expresses the curve of the control input $u$. Figure 4 express the curve the tracking error of the proposed control method. Figure 4 reveals that the evolution of the proposed adaptive controller remains within the prescribed performance bounds for all $t \geq 0$; that is, the prescribed performance is satisfied.

\section{Conclusion}

In this paper, fuzzy adaptive output feedback tracking control problem has been investigated for a class of nonlinear stochastic systems in strict-feedback form. The addressed stochastic nonlinear systems contain unknown nonlinear

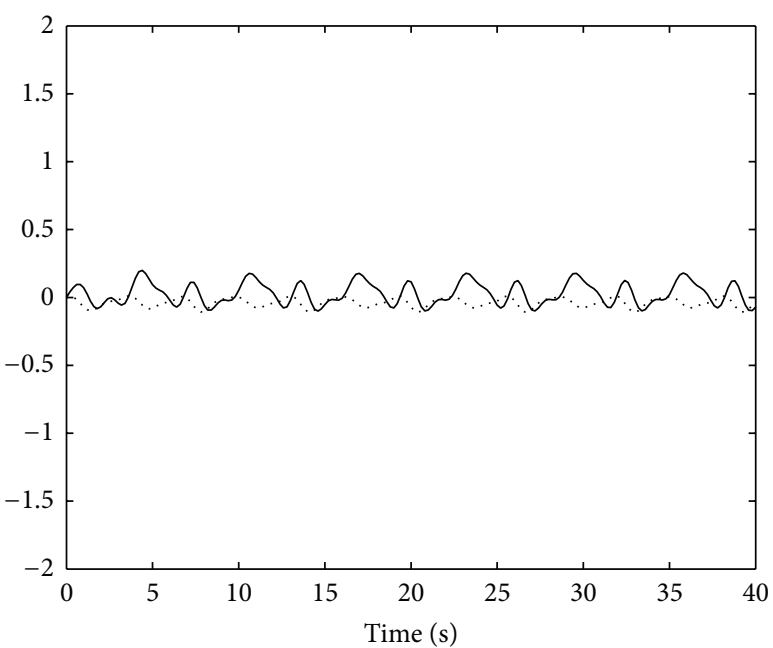

Figure 2: The curves of $e_{1}$ (solid line) and $e_{2}$ (dot line).

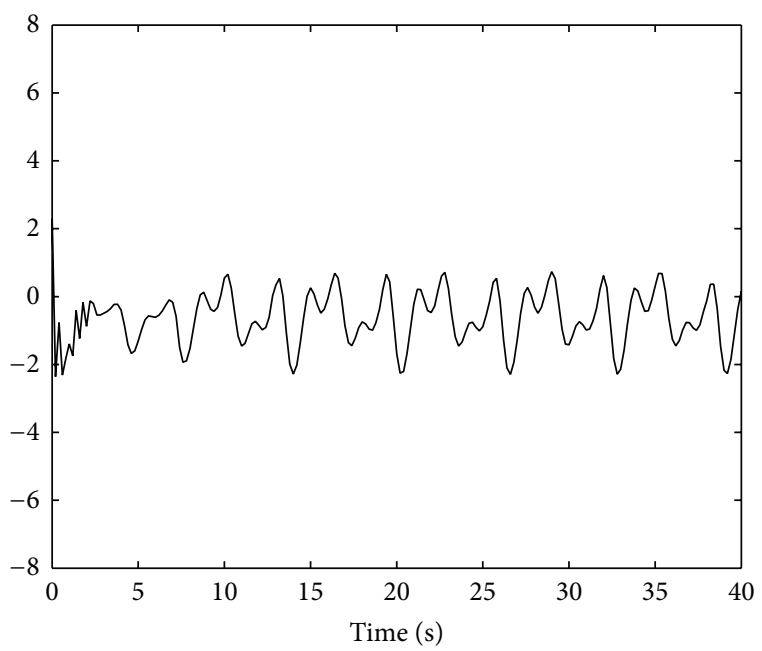

Figure 3: The curve of $u$.

functions and without the measurements of the states. Fuzzy logic systems are used to identify the unknown nonlinear functions, and a fuzzy state filter observer has been designed for estimating the unmeasured states. By applying 


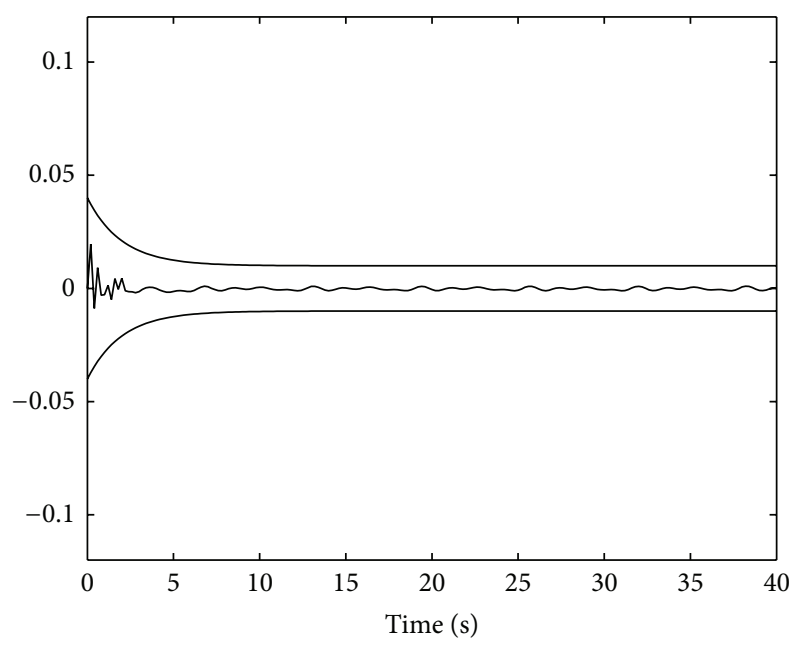

FIGURE 4: The curves of $z_{1}$ and performance bounds.

the backstepping recursive design technique and the predefined performance technique, a new robust fuzzy adaptive output-feedback control approach has been developed, and the stability of the closed-loop system has been proved. The main advantages of the proposed control approach are that it cannot only solve the state unmeasured problem of nonlinear stochastic systems, but can also guarantee that the tracking error converges to an adjustable neighborhood of the origin and remains within the prescribed performance bounds. Future research will be concentrated on an adaptive fuzzy output-feedback tracking control for multiinput and multioutput stochastic nonlinear systems with unmeasured states based on the results of $[22,23]$ and this paper.

\section{Conflict of Interests}

None of the authors of the paper have declared any conflict of interests.

\section{Acknowledgments}

This work was supported by the National Natural Science Foundation of China (nos. 61374113, 61074014, and 61203008) and Liaoning Innovative Research Team in University (LT2012013).

\section{References}

[1] Y. G. Niu, W. C. H. Daniel, and J. Lam, "Robust integral sliding mode control for uncertain stochastic systems with time-varying delay," Automatica, vol. 41, no. 5, pp. 873-880, 2005.

[2] P. Shi, Y. Q. Xia, G. P. Liu, and D. Rees, "On designing of slidingmode control for stochastic jump systems," IEEE Transactions on Automatic Control, vol. 51, no. 1, pp. 97-103, 2006.

[3] M. Liu, D. W. C. Ho, and Y. G. Niu, "Stabilization of Markovian jump linear system over networks with random communication delay," Automatica, vol. 45, no. 2, pp. 416-421, 2009.
[4] H. Y. Li, C. Wang, P. Shi, and H. Gao, "New passivity results for uncertain discrete-time stochastic neural networks with mixed time delays," Neurocomputing, vol. 73, no. 16-18, pp. 3291-3299, 2010.

[5] Z. G. Pan and T. Başar, "Adaptive controller design for tracking and disturbance attenuation in parametric strict-feedback nonlinear systems," IEEE Transactions on Automatic Control, vol. 43, no. 8, pp. 1066-1083, 1998.

[6] H. Deng and M. Krstic, "Output-feedback stochastic nonlinear stabilization," IEEE Transactions on Automatic Control, vol. 44, no. 2, pp. 328-333, 1999.

[7] Z. Wu, X. Xie, P. Shi, and Y.-q. Xia, "Backstepping controller design for a class of stochastic nonlinear systems with Markovian switching," Automatica, vol. 45, no. 4, pp. 997-1004, 2009.

[8] Y. Xia, M. Fu, P. Shi, Z. Wu, and J. Zhang, "Adaptive backstepping controller design for stochastic jump systems," IEEE Transactions on Automatic Control, vol. 54, no. 12, pp. 28532859, 2009.

[9] H. B. Ji and H. S. Xi, "Adaptive output-feedback tracking of stochastic nonlinear systems," IEEE Transactions on Automatic Control, vol. 51, no. 2, pp. 355-360, 2006.

[10] S. J. Liu, J. F. Zhang, and Z. P. Jiang, "Decentralized adaptive output-feedback stabilization for large-scale stochastic nonlinear systems," Automatica, vol. 43, no. 2, pp. 238-251, 2007.

[11] S. J. Liu, S. S. Ge, and J. F. Zhang, "Adaptive output-feedback control for a class of uncertain stochastic non-linear systems with time delays," International Journal of Control, vol. 81, no. 8, pp. 1210-1220, 2008.

[12] W. S. Chen, L. C. Jiao, J. Li, and R. Li, "Adaptive NN backstepping output-feedback control for stochastic nonlinear strictfeedback systems with time-varying delays," IEEE Transactions on Systems, Man, and Cybernetics B, vol. 40, no. 3, pp. 939-950, 2010.

[13] J. Li, W. S. Chen, J. M. Li, and Y. Q. Fang, "Adaptive NN outputfeedback stabilization for a class of stochastic nonlinear strictfeedback systems," ISA Transactions, vol. 48, no. 4, pp. 468-475, 2009.

[14] J. Li, W. S. Chen, and J. M. Li, "Adaptive NN output-feedback decentralized stabilization for a class of large-scale stochastic nonlinear strict-feedback systems," International Journal of Robust and Nonlinear Control, vol. 21, no. 4, pp. 452-472, 2011.

[15] Q. Zhou, P. Shi, H. H. Liu, and S. Y. Xu, "Neural-network-based decentralized adaptive output-feedback control for large-scale stochastic nonlinear systems," IEEE Transactions on Systems, Man, and Cybernetics B, vol. 42, no. 6, pp. 1608-1619, 2012.

[16] S. C. Tong, Y. Li, Y. M. Li, and Y. Liu, "Observer-based adaptive fuzzy backstepping control for a class of stochastic nonlinear strict-feedback systems," IEEE Transactions on Systems, Man, and Cybernetics B, vol. 41, no. 6, pp. 1693-1704, 2011.

[17] S. C. Tong, T. Wang, Y. M. Li, and B. Chen, "A combined backstepping and stochastic small-gain approach to robust adaptive fuzzy output feedback control," IEEE Transactions on Fuzzy Systems, vol. 21, no. 2, pp. 314-327, 2013.

[18] S. C. Tong, Y. M. Li, and T. Wang, "Adaptive fuzzy decentralized output feedback control for stochastic nonlinear large-scale systems using DSC technique," International Journal of Robust and Nonlinear Control, vol. 23, no. 4, pp. 381-399, 2013.

[19] C. P. Bechlioulis and G. A. Rovithakis, "Robust adaptive control of feedback linearizable MIMO nonlinear systems with prescribed performance," IEEE Transactions on Automatic Control, vol. 53, no. 9, pp. 2090-2099, 2008. 
[20] C. P. Bechlioulis and G. A. Rovithakis, "Prescribed performance adaptive control for multi-input multi-output affine in the control nonlinear systems," IEEE Transactions on Automatic Control, vol. 55, no. 5, pp. 1220-1226, 2010.

[21] L. X. Wang, Adaptive Fuzzy Systems and Control: Design and Stability Analysis, Prentice Hall, Englewood Cliffs, NJ, USA, 1994.

[22] H. Y. Li, X. J. Jing, H. K. Lam, and P. Shi, "Fuzzy sampleddata control for uncertain vehicle suspension systems," IEEE Transactions on Cybernetics, no. 99, 1 page, 2013.

[23] H. Y. Li, X. J. Jing, and H. R. Karimi, "Output-feedback based $H_{\infty}$ control for active suspension systems with control delay," IEEE Transactions on Industrial Electronics, vol. 61, no. 1, pp. 436-446, 2014. 


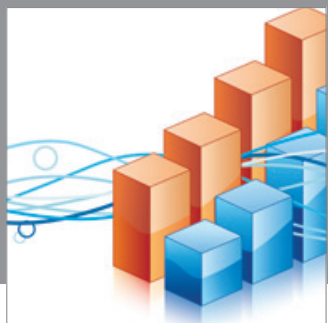

Advances in

Operations Research

mansans

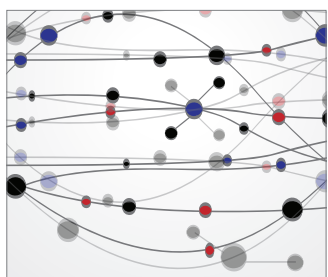

The Scientific World Journal
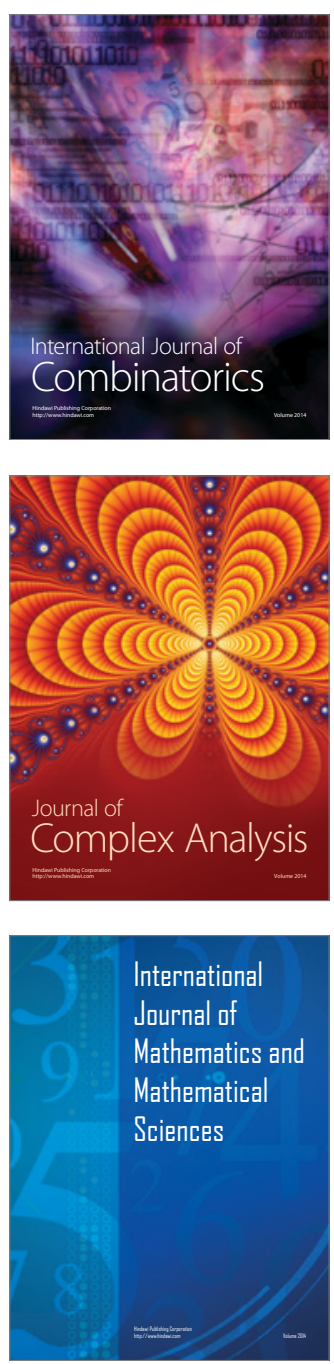
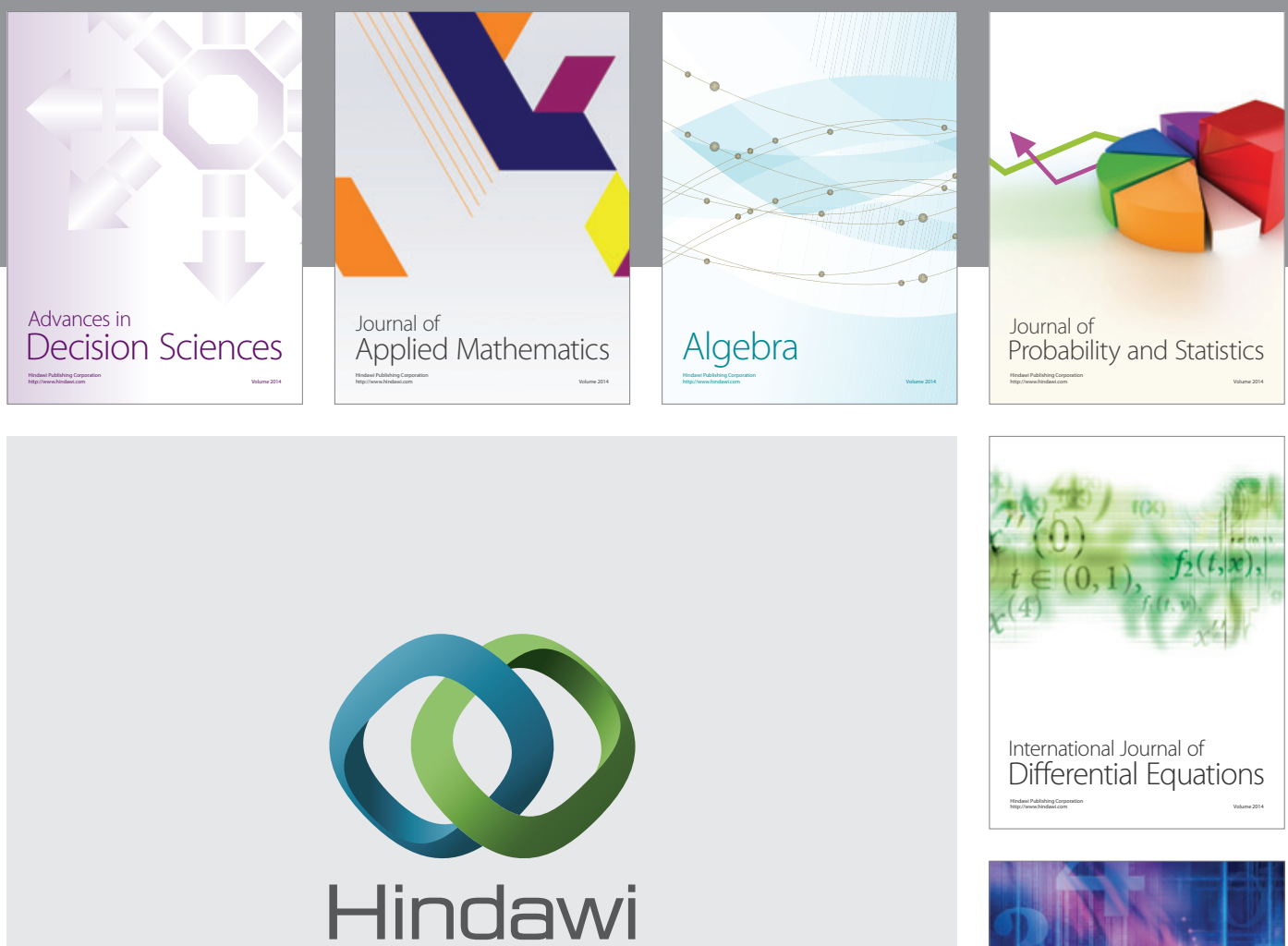

Submit your manuscripts at http://www.hindawi.com
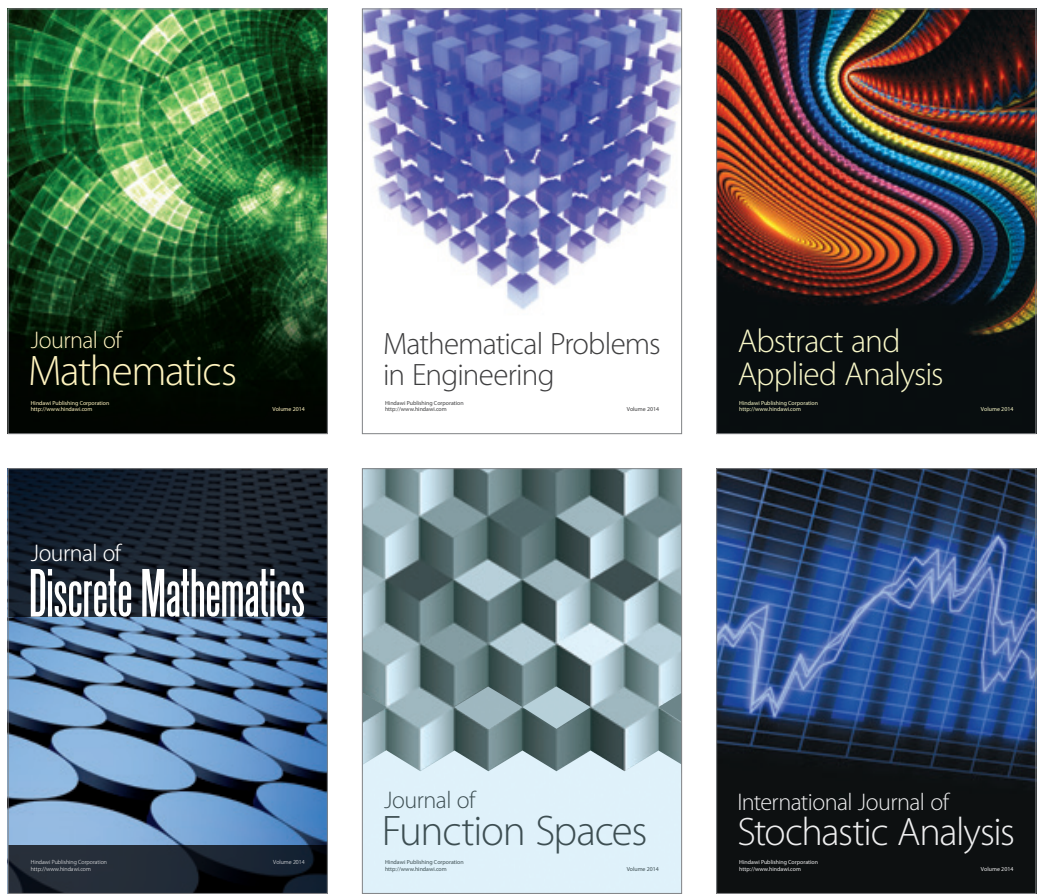

Journal of

Function Spaces

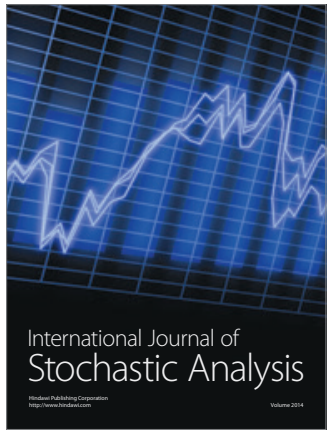

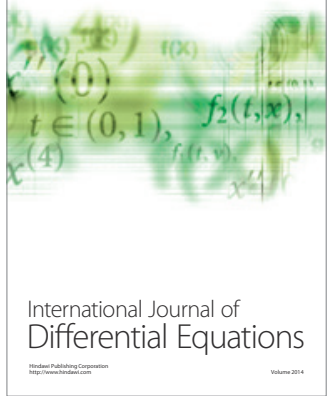
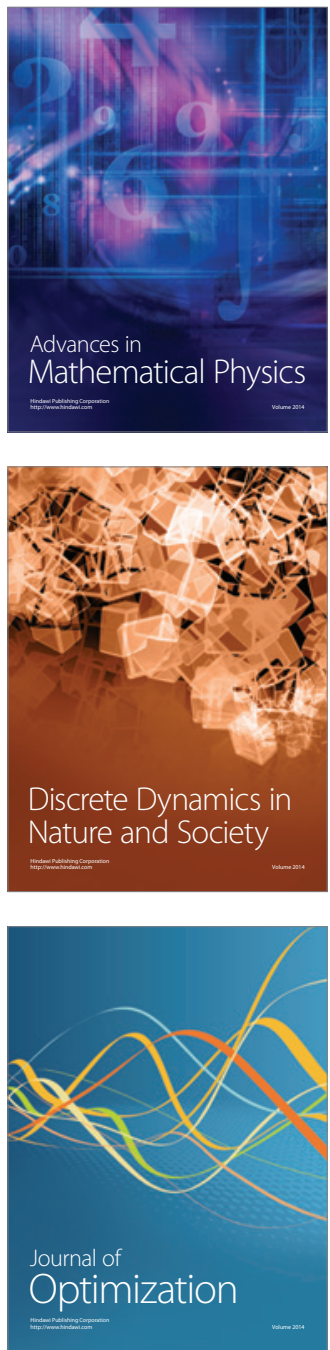